\title{
Effects of dietary extra-virgin olive oil on behaviour and brain biochemical parameters in ageing rats
}

\author{
Vanessa Pitozzi ${ }^{1}$, Michela Jacomelli ${ }^{1}$, Mohamed Zaid ${ }^{1}$, Cristina Luceri ${ }^{1}$, Elisabetta Bigagli ${ }^{1}$, \\ Maura Lodovici ${ }^{1}$, Carla Ghelardini ${ }^{1}$, Elisa Vivoli ${ }^{1}$, Monica Norcini ${ }^{1}$, Marco Gianfriddo ${ }^{2}$, \\ Sonia Esposto $^{3}$, Maurizio Servili ${ }^{3}$, Guido Morozzi ${ }^{4}$, Elisabetta Baldi ${ }^{5}$, Corrado Bucherelli ${ }^{5}$, \\ Piero Dolara ${ }^{1}$ and Lisa Giovannelli ${ }^{1}{ }^{*}$ \\ ${ }^{1}$ Department of Preclinical and Clinical Pharmacology, University of Florence, Viale G. Pieraccini 6, 50139 Florence, Italy \\ ${ }^{2}$ Siena Biotech Spa, Via Fiorentina 1, 53100 Siena, Italy \\ ${ }^{3}$ Department of Food Sciences, University of Perugia, Via S. Costanzo, 06126 Perugia, Italy \\ ${ }^{4}$ Department of Public Health, University of Perugia, Via del Giochetto, 06126 Perugia, Italy \\ ${ }^{5}$ Department of Physiology, University of Florence, Viale Morgagni 63, 50139 Florence, Italy
}

(Received 30 July 2009 - Revised 6 October 2009 - Accepted 3 December 2009 - First published online 14 January 2010)

\begin{abstract}
The aim of the present study was to verify whether extra-virgin olive oil, a dietary component naturally containing phenolic antioxidants, has the potential to protect the brain from the deleterious effects of ageing. To accomplish this goal, we used male rats fed a high-energy diet containing either maize oil, or extra-virgin olive oil with high or low phenol content ( 720 or $10 \mathrm{mg}$ total phenols $/ \mathrm{kg}$ oil, corresponding to a daily dose of 4 or $0.05 \mathrm{mg}$ total phenols $/ \mathrm{kg}$ body weight, respectively) from age 12 months to senescence. The measured endpoints were biochemical parameters related to oxidative stress and functional tests to evaluate motor, cognitive and emotional behaviour. Olive oil phenols did not exert major protective actions on motor and cognitive function, as we observed only a tendency to improved motor coordination on the rotarod in the old animals treated with the oil rich in phenols ( $40 \%$ average increase in the time to first fall; $P=0 \cdot 18$ ). However, an interesting finding of the present study was a reduced step-through latency in the light-dark box test, found in the older animals upon treatment with the oil rich in antioxidant phenols, possibly indicating an anxiety-lowering effect. This effect was associated with decreased glutathione reductase activity and expression in the brain, a phenomenon previously associated with decreased anxiety in rodents. These results indicate a previously undetected effect of a diet containing an olive oil rich in phenols. Further studies are warranted to verify whether specific food antioxidants might also have an effect on emotional behaviour.
\end{abstract}

Olive oil phenols: Oxidative damage: Anxiety: Glutathione reductase

Ageing is a complex phenomenon, leading to the progressive impairment of a number of metabolic and physiological functions. The free radical theory of ageing is still one of the mainstream hypotheses to explain the mechanisms underlying this condition, particularly in the brain. Alterations in oxidation parameters have been repeatedly reported in the ageing brain, such as increased nuclear and mitochondrial DNA oxidation ${ }^{(1,2)}$, increased protein carbonylation ${ }^{(3)}$, changed activity of antioxidant and mitochondrial enzymes and reduced levels of endogenous scavengers ${ }^{(3,4)}$.

Oxidative stress is also thought to play a role in agerelated neurological conditions such as Alzheimer's and Parkinson's diseases ${ }^{(5,6)}$. Thus, it is possible, at least in theory, that age-related brain dysfunctions can be ameliorated by restoring the altered redox equilibrium and decreasing oxidative damage.
There is growing interest in finding dietary strategies capable of protecting from the deleterious effects of ageing on the brain, and antioxidants have been widely investigated in this respect. Among these, flavonoids have shown neuroprotective potential both in vitro and in vivo ${ }^{(7)}$. Diets rich in fresh fruit and vegetables counteract some age-related biochemical, electrophysiological and behavioural deficits in rats ${ }^{(8,9)}$. Long-term dietary administration of thiol-containing antioxidants has been shown to prevent glutathione and mitochondrial DNA oxidation and the loss of motor coordination in aged mice ${ }^{(10)}$.

In the present study we chose to focus on olive oil antioxidants. Olive oil is a typical component of the Mediterranean diet, containing variable amounts of phenolic antioxidants ${ }^{(11)}$. Among this group of phenols, hydroxytyrosol (3,4-DHPEA) and its derivatives are mainly responsible for the antioxidant

\footnotetext{
Abbreviations: 3,4-DHPEA, hydroxytyrosol; FPG, formamidopyrimidine glycosylase; GPx, glutathione peroxidase; GR, glutathione reductase; GSH, reduced glutathione; GSSG, oxidised glutathione; H-EVOO, extra-virgin olive oil rich in natural antioxidants; L-EVOO, extra-virgin olive oil poor in natural antioxidants; MO, maize oil; SOD, superoxide dismutase; XO, xanthine oxidase.

*Corresponding author: Dr L. Giovannelli, fax +39055 4271 280, email lisa.giovannelli@unifi.it
} 
activity of olive oil ${ }^{(12)}$. Orally administered 3,4-DHPEA is absorbed in rats and human subjects ${ }^{(13,14)}$ and has shown antioxidant activity both in vitro ${ }^{(15,16)}$ and in vivo ${ }^{(13,17)}$. The ester of 3,4-DHPEA with elenoic acid (oleuropein) is also a potent antioxidant active in the micromolar range, and both oleuropein and 3,4-DHPEA have been proven to be more potent antioxidants than vitamin $\mathrm{E}^{(18)}$. Furthermore, being amphipathic molecules, these compounds can be anticipated to easily pass the blood-brain barrier. Ex vivo data have provided evidence of a neuroprotective activity of olive oil phenols upon oral administration in mice ${ }^{(19)}$. The fatty acid component of olive oil, represented mainly by monounsaturated oleic acid, might also have a beneficial impact on ageing. A high MUFA intake appeared to be protective against age-related cognitive decline in one human epidemiological study ${ }^{(20)}$.

We have previously shown that long-term treatment with extra-virgin olive oil with high phenolic antioxidant content was associated with some beneficial health effects in ageing rats, such as delayed mortality, reduced incidence of ulcerative dermatitis, and decreased oxidative damage in blood ${ }^{(21)}$. We selected an extra-virgin Italian olive oil naturally rich in phenolic antioxidants and administered it to rats from middle to advanced age. The oil was mixed with the animal diet to reach a final fat proportion of $23 \%$ (w/w, dry diet), corresponding to about $44 \%$ of the energy provided by the whole diet, to mimic the total lipid contribution common in the diet of humans living in Western Europe and North America $^{(22)}$. As a control, an identical olive oil with a lower content of antioxidants was used. We also administered maize oil as an additional control to check for the effects of different antioxidants and fatty acids. The measured endpoints were a series of biochemical parameters related to oxidative stress in the brain and functional tests to evaluate motor, cognitive, emotional behaviour and pain sensitivity.

\section{Methods and materials}

\section{Animals and treatments}

All procedures were carried out in agreement with the European Union Regulations on the Care and Use of Laboratory Animals (OJ of ECL 358/1, 12/18/1986) and the experiments were conducted according to Italian regulations on the protection of animals used for experimental and other scientific purposes (DM 116/1992), after approval from the Italian Ministry for Scientific Research.

Male Wistar rats aged 10 months at the beginning of the experiment were used. The animals were randomly subdivided into three groups of twenty rats each and fed different diets ( $15 \mathrm{~g} / \mathrm{rat}$ per $\mathrm{d})$ for 12 months. The experimental diets were prepared using components purchased from Piccioni (Gessate, Milan, Italy). Fresh diet was put in the trough every day. We used a modified high-fat American Institute of Nutrition (AIN)-76 diet (lipids providing about $40 \%$ of the total energy), composed of ( $\mathrm{g} / 100 \mathrm{~g}$ diet): 32.2 sucrose, 23.0 fat, $23 \cdot 0$ casein, $10 \cdot 1$ maize starch, $6 \cdot 0$ cellulose, 4.0 AIN-76 Mineral mix, 1.2 AIN-76 Vitamin mix, 0.3 methionine and $0 \cdot 2$ choline. Dietary components, except for the oils, were purchased from Piccioni (Gessate, Milan, Italy).
One group of rats was fed a diet in which the lipid component was provided by maize oil (MO), another group by an extra-virgin olive oil high in natural antioxidants (H-EVOO), and the third group by an extra-virgin olive oil with low amounts of phenolic compounds (L-EVOO). MO was purchased from Tampieri S.p.A. (Faenza, Ravenna, Italy), while H-EVOO was purchased from Cipolloni S.p.A. and Petesse S.p.A. (Foligno, Perugia, Italy). The L-EVOO was obtained from $\mathrm{H}-\mathrm{EVOO}$ as follows: the extra-virgin oil was homogenised for $1 \mathrm{~min}$ with water $(1: 1, \mathrm{v} / \mathrm{v})$, and the oil was separated by centrifugation (Westfalia separator); this procedure was repeated six times. Then the oil was filtered through a cellulose acetate membrane.

During the 12-month treatment with the different oils, the animals were checked for their general health status every day and body weight was measured every 2 weeks. After decapitation, blood was collected by decanting into anticoagulant tubes (containing sodium citrate) and centrifuged at $1000 \mathrm{~g}$ for $10 \mathrm{~min}$ to separate plasma from erythrocytes. Erythrocyte samples were stored at $-80^{\circ} \mathrm{C}$.

The brain was removed from the killed animals and weighed. Part of the tissue was used immediately to measure the endogenous levels of DNA damage and lipid peroxidation, part was stored at $-80^{\circ} \mathrm{C}$ and part was stored in RNA later buffer at $-20^{\circ} \mathrm{C}$ for RT-PCR (see below).

\section{Extraction and HPLC analysis of phenolic compounds of olive oils}

The extraction of H-EVOO and L-EVOO phenols and the HPLC analysis were conducted as reported ${ }^{(23)}$, using an Agilent Technologies system model 1100 (Agilent Technologies, Palo Alto, CA, USA), composed of a vacuum degasser, a quaternary pump, an autosampler, a thermostated column compartment, a diode array detector and a fluorescence detector. The analysis of the oil extract was performed using Spherisorb ODS-1 C18 columns $(250 \times 4.6 \mathrm{~mm})$ with a particle size of $5 \mu \mathrm{m}$ (Phase Separation Ltd, Deeside, Clwyd, UK). The mobile phase was composed of $0.2 \%$ acetic acid ( $\mathrm{pH} 3 \cdot 1)$ in water (solvent $\mathrm{A}) /$ methanol (solvent $\mathrm{B}$ ) at a flow rate of $1 \mathrm{ml} / \mathrm{min}$, and the gradient was changed as follows: $95 \% \mathrm{~A} / 5 \% \mathrm{~B}$ for $2 \mathrm{~min}, 75 \% \mathrm{~A} / 25 \% \mathrm{~B}$ for $8 \mathrm{~min}$, $60 \% \mathrm{~A} / 40 \% \mathrm{~B}$ for $10 \mathrm{~min}, 50 \% \mathrm{~A} / 50 \% \mathrm{~B}$ for $16 \mathrm{~min}, 0 \%$ $\mathrm{A} / 100 \% \mathrm{~B}$ for $14 \mathrm{~min}$ and this composition was maintained for $10 \mathrm{~min}$. Then, the initial conditions were reset and equilibrium was reached for $13 \mathrm{~min}$; the total running time was $73 \mathrm{~min}$.

\section{Extraction and HPLC analysis of $\alpha$-tocopherol from the olive oils}

The $\alpha$-tocopherol was analysed as described ${ }^{(24)}: 2 \mathrm{~g}$ of oil were dissolved in $10 \mathrm{ml}$ hexane and then filtered through a polyvinyledene diflouride (PVDF) syringe filter $(0 \cdot 2 \mu \mathrm{m})$ and injected into the HPLC system through a Waters $\mu$ Porasil column $(300 \mathrm{~mm} \times 3.9 \mathrm{~mm} \times 10 \mu \mathrm{m}$; Waters, Milford, MA, USA) using a $100 \%$ step mobile-phase gradient of $n$-hexane with $0.5 \%$ isopropylic alcohol (A)/0\% $n$-hexane with $10 \%$ isopropylic alcohol (B) for $4 \mathrm{~min}, 60 \% \mathrm{~A} / 40 \% \mathrm{~B}$ for $14 \mathrm{~min}, 40 \% \mathrm{~A} / 60 \% \mathrm{~B}$ for $4 \mathrm{~min}, 100 \% \mathrm{~A} / 0 \% \mathrm{~B}$ for $3 \mathrm{~min}$. This last gradient was maintained for $5 \mathrm{~min}$ with a flow rate 
of $2 \mathrm{ml} / \mathrm{min}$. The total running time was $30 \mathrm{~min}$ and the injection volume $50 \mu \mathrm{l}$. The detector was a fluorescence detector operated at an excitation wavelength set at $294 \mathrm{~nm}$ and the emission at $330 \mathrm{~nm}$.

\section{Behavioural tests}

All the tests were performed in an acoustically isolated room, kept at $20 \pm 1^{\circ} \mathrm{C}$, starting at about 09.00 hours. Illumination inside the room was 60 lux. All measurements were done blindly by the operators.

Exploratory activity: hole board test. The hole board test consisted of a $40 \mathrm{~cm}^{2}$ plane with sixteen flush-mounted cylindrical holes $(3 \mathrm{~cm}$ diameter) distributed four by four in an equidistant, grid-like manner (Ugo Basile, Varese, Italy). Rats were placed on the centre of the board one by one and allowed to move about freely for a period of $5 \mathrm{~min}$ each. Two electric eyes, crossing the plane from midpoint to midpoint of opposite sides, thus dividing the plane into four equal quadrants, automatically signalled the movement of the animal (counts in $5 \mathrm{~min}$ ) on the surface of the plane (spontaneous motility). Miniature photoelectric cells in each of the sixteen holes recorded (counts in $5 \mathrm{~min}$ ) the exploration of the holes (exploratory activity) by the rats. Ten rats per group were tested.

Exploratory activity: multiple Y-maze. This test was performed using an apparatus previously described by Ambrogi Lorenzini et al. ${ }^{(25)}$. The rats were left free to explore the maze without being subjected to punishments or rewards for a total time of $3 \mathrm{~min}$. The space travelled by each animal during this time was measured by evaluating the number of visited arms, and expressed in $\mathrm{cm}$.

Motor coordination: rotarod. The rats were tested at 19 months of age (treatment week 38). On day 1 the rats were given a habituation trial where they were placed on the rotarod apparatus (Ugo Basile) initially at speed 0 (30s) and then at a constant speed $(4 \mathrm{rpm})$. The rats had to remain on the rotarod for $60 \mathrm{~s}$. The following day each rat was given two trials during which the rotarod started at $4 \mathrm{rpm}$ and accelerated to $20 \mathrm{rpm}$ over a period of $90 \mathrm{~s}$. The maximum trial length was 3 min and there was a 15 min rest period between each trial.

Pain sensitivity: Randall and Selitto paw-pressure test. The nociceptive threshold in the rat was determined with an analgesimeter (Ugo Basile), according to the method described by Leighton et al. ${ }^{(26)}$. The stimulus at which rats withdrew the paw was evaluated at different times during the treatment. Results represent the mean of mechanical thresholds expressed as grams. To avoid any possible damage to the animal paw the maximum applied force was fixed at $240 \mathrm{~g}$.

Memory: social learning test. The social learning test was performed according to Mondadori et al. ${ }^{(27)}$. Juvenile male rats $(90-110 \mathrm{~g})$ were used as social stimuli for studying adult and old rats. On the first day of the experiment, a juvenile rat was introduced into the adult male's cage and the time spent in social-investigatory behaviour by the adult male within a 5 min fixed interval was recorded. After $24 \mathrm{~h}$, the same juvenile rat was placed again into the mature male's cage and social-investigatory behaviour was recorded during a $5 \mathrm{~min}$ interval. On the same day, the social-investigatory behaviour towards a second unfamiliar juvenile rat was also recorded.
Memory: fear conditioning test. A basic Skinner box module was employed to induce fear conditioning ${ }^{(28)}$. The floor was made of stainless-steel rods connected to a shock delivery apparatus (Grid Floor Shocker, model E13-08; Coulbourn Instruments, Whitehall, PA, USA). The apparatus was connected to a stimulus programming device (Scatola di commando (control box) Arco 2340; Ugo Basile) in order to predetermine number, duration and rate of electric stimuli. On day 1 the rat was taken from the home cage, and carried to a soundproof room. Once there, it was placed inside the conditioning apparatus. The rat was left undisturbed for $3 \mathrm{~min}$. After this time, a series of seven electric shocks of $1 \mathrm{~s}$ each (alternate current duration $20 \mathrm{~ms}$, frequency $20 \mathrm{~ms}$, intensity $100 \mathrm{~V}$ ) at $30 \mathrm{~s}$ intervals was administered through the grid. The rat was left undisturbed for 2 min after the end of the stimulation pattern, and brought back to the home cage immediately thereafter. Context freezing response was measured in the same apparatus used for conditioning. Each rat was placed again in the apparatus on days 3, 6, 10 and 13 after the conditioning session, and left undisturbed for 6 min. During this time, the total accumulated freezing time (i.e. total seconds spent freezing) was measured. Freezing (immobility) was defined as the complete absence of somatic motility except for respiratory movements.

Anxiety: light-dark preference. We used a 'light-dark box' built in our department ${ }^{(29)}$, made of two chambers of equal dimensions, one of white opaque plastic, and the other of black opaque plastic. The floors of both chambers were made of stainless-steel rods. The two chambers were separated by a guillotine door. The animals were taken singly from their home cages and brought to the adjacent acoustically insulated room, where the light-dark box apparatus was placed. Each rat was placed in the lighted chamber, facing away from the closed connecting door. The guillotine door was raised $5 \mathrm{~s}$ later. The time spent by the rat in the lighted chamber before going into the dark chamber with all four paws (stepthrough latency) was measured. The total time spent in the light or in the dark compartment and the number of crossings between the two compartments over a period of $3 \mathrm{~min}$ were also measured.

\section{Measurement of oxidative damage-related parameters}

Comet assay. DNA strand breaks and oxidative damage in cerebral cortex homogenates were evaluated by the single cell-gel electrophoresis (comet assay) as previously described $^{(2)}$. To determine oxidised DNA bases, the bacterial repair enzyme, formamidopyrimidine glycosylase (FPG), which introduces breaks at purine oxidation sites, was used. Data were expressed as percentage of total fluorescence migrated to the tail of each nucleus (\% DNA in tail), a parameter linearly related to the number of DNA breaks. The value of \% DNA in tail obtained without FPG incubation estimated the basal number of DNA strand breaks, whereas specific oxidative damage on purines (FPG-sensitive sites) was assessed by subtracting the basal value from that obtained with FPG.

Determination of malonyldialdehyde. Malonyldialdehyde was determined after derivatisation with di- $n$-hexyl phthalate as described by Mateos et al. ${ }^{(30)}$. Briefly, brain homogenates $(100 \mathrm{mg}$ tissue in $1 \mathrm{ml}$ Tris-base buffer containing 
$0 \cdot 2 \mathrm{M}$-sucrose and $5 \mathrm{~mm}$-dithiothreitol, $\mathrm{pH} 7 \cdot 4)$ were centrifuged at $10000 \mathrm{~g}$ for $30 \mathrm{~min}$ at $4^{\circ} \mathrm{C}$. Samples of $250 \mu \mathrm{l}$ of the supernatant fraction were used, and alkaline hydrolysis of protein-bound malonyldialdehyde was achieved by adding $50 \mu \mathrm{l}$ of $6 \mathrm{M}-\mathrm{NaOH}$ to each sample and incubating this mixture in a $60^{\circ} \mathrm{C}$ water-bath for $30 \mathrm{~min}$. Proteins were then precipitated with $250 \mu \mathrm{l}$ of $30 \%$ (v/v) TCA. A $250 \mu \mathrm{l}$ volume of supernatant fraction was mixed with $25 \mu$ l of di- $n$-hexyl phthalate prepared as a $5 \mathrm{~mm}$ solution in $2 \mathrm{M}$-hydrochloric acid. Finally, this reaction mixture was incubated for $30 \mathrm{~min}$ at room temperature in the dark. A sample of $100 \mu \mathrm{l}$ of the mixture was injected into a Shimadzu LC-10AD HPLC system with a Waters Spherisorb RP-18 column $(4.6 \mathrm{~mm} \times 150 \mathrm{~mm}$, ODS $25 \mu \mathrm{m}$; Supelco, Sigma-Aldrich, Milan, Italy). Samples were isocratically eluted with a mixture of $0.2 \%(\mathrm{v} / \mathrm{v})$ acetic acid in deionised water, and acetonitrile $(62: 38, \mathrm{v} / \mathrm{v})$ at a flow rate of $0.8 \mathrm{ml} / \mathrm{min}$ at room temperature. Chromatograms were acquired at $310 \mathrm{~nm}$. The calibration curve was prepared by diluting a standard solution of tetraethoxypropane. Data were expressed as nmol malonyldialdehyde/mg protein.

Measurement of reduced and oxidised glutathione levels. About $100 \mathrm{mg}$ of cerebral cortex samples were homogenised in $50 \mathrm{~mm}$-phosphate buffer $(\mathrm{pH} 6.5)$, containing $1 \mathrm{mM}$ EDTA, and centrifuged at $10000 \mathrm{~g}$ for $15 \mathrm{~min}$ at $4^{\circ} \mathrm{C}$. The determination of reduced glutathione $(\mathrm{GSH})$ and oxidised glutathione (GSSG) concentration was performed using an assay kit of Cayman Chemical Company (Ann Arbor, MI, USA) and a microplate reader (Wallac VICTOR ${ }^{\mathrm{TM}} 1420$ Multilabel Counter; Perkin Elmer, Waltham, MA, USA). The method was based on the reaction of the sulfhydryl group of GSH with 5,5'-dithiobis-2-nitrobenzoic acid (Ellman's reagent) leading to the formation of yellowcoloured 5-thio-2-nitrobenzoic acid (TNB). The rate of TNB production was directly proportional to the concentration of GSH in the sample. Deproteinisation of samples (with $10 \%$ metaphosphoric acid and $4 \mathrm{M}$-triethanolamine) and preparation for exclusive measurement of GSSG (with $1 \mathrm{M}$-2-vinylpyridine in ethanol) were performed in accordance with Cayman recommendations. The levels of GSH and GSSG were then calculated per mg protein of samples.

Measurement of enzyme activity. For glutathione peroxidase (GPx) and glutathione reductase (GR) activity measurement, $100 \mathrm{mg}$ of cerebral cortex samples were homogenised

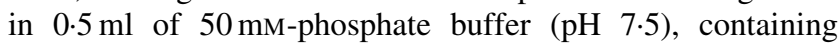
$1 \mathrm{mM}-\mathrm{EDTA}$ and $1 \%$ protease inhibitor, and centrifuged for $15 \mathrm{~min}$ at $10000 \mathrm{~g}$ at $4^{\circ} \mathrm{C}$. For superoxide dismutase (SOD) and xanthine oxidase (XO) activity, $100 \mathrm{mg}$ of cerebral cortex were homogenised in $1 \mathrm{ml}$ of $50 \mathrm{~mm}$-PBS and centrifuged at $1500 \mathrm{~g}$ for $15 \mathrm{~min}$ at $4^{\circ} \mathrm{C}$. The resulting supernatant fractions were used to measure all enzymic activities.

Measurement of enzyme activity: glutathione peroxidase. GPx activity was measured using the method of Paglia \& Valentine $^{(31)}$, modified to suit a ninety-six-well plate format, by a coupled reaction with the oxidation of NADPH by GR. The assay mixture consisted of $50 \mathrm{~mm}$-phosphate buffer (pH 7.4), 5 mM-EDTA, 2 mM-GSH, GR (2 U/ml) and $0.5 \mathrm{~mm}-$ NADPH. The reaction was initiated by the simultaneous addition of $20 \mu \mathrm{l}$ of supernatant fraction and $1 \mathrm{mM}$-cumene hydroperoxide. The samples were assayed in duplicate, and the decrease in absorbance was followed for $5 \mathrm{~min}$ at $340 \mathrm{~nm}$, using a microplate reader (Wallac VICTOR ${ }^{\mathrm{TM}} 1420$ Multilabel Counter; Perkin Elmer, Waltham, MA, USA). GPx activity was defined as nmol oxidised NADPH/min at $25^{\circ} \mathrm{C}$.

Measurement of enzyme activity: glutathione reductase. GR activity was measured by the method of Carlberg \& Mannervik ${ }^{(32)}$, modified to suit a ninety-six-well plate format. A volume of $20 \mu$ l of supernatant fraction, diluted $1: 10$, was added to a mixture containing $50 \mathrm{~mm}$-phosphate buffer (pH 7.5), 1 mM-EDTA and $1 \mathrm{mm-GSSG.} \mathrm{The} \mathrm{absor-}$ bance was followed at $340 \mathrm{~nm}$ for $5 \mathrm{~min}$, following initiation of the reaction by the addition of $0.2 \mathrm{~mm}-\mathrm{NADPH}$. The samples were assayed in duplicate and GR activity was defined as nmol oxidised NADPH/min at $25^{\circ} \mathrm{C}$.

Measurement of enzyme activity: superoxide dismutase. A sample of $100 \mu \mathrm{l}$ of 1:10 diluted cortex supernatant fraction was used to determine total SOD activity by nitro blue tetrazolium reaction: $1.2 \mathrm{ml}$ of $50 \mathrm{mM}-\mathrm{Na}_{2} \mathrm{CO}_{3}(\mathrm{pH} \mathrm{10.2)}, 50 \mu \mathrm{l}$ of $3 \mathrm{~mm}$-EDTA, $50 \mu \mathrm{l}$ of $3 \mathrm{~mm}$-xanthine, $50 \mu \mathrm{l}$ of $0.8 \mathrm{~mm}$ nitro blue tetrazolium and $50 \mu \mathrm{l}$ of $\mathrm{XO}(140 \mathrm{mU} / \mathrm{ml}$ initial concentration) were added to each sample. Samples were incubated for $30 \mathrm{~min}$ at $24^{\circ} \mathrm{C}$. The inhibition of nitro blue tetrazolium reduction in each sample was determined spectrophotometrically at $560 \mathrm{~nm}$. SOD activity, expressed as U/mg protein, was evaluated by referring to inhibition brought about by the SOD standard measured at the same time.

Measurement of enzyme activity: xanthine oxidase. A further $100 \mu \mathrm{l}$ fraction of the diluted (1:10) cortex supernatant fraction was used for assaying XO activity. Briefly, $1.3 \mathrm{ml}$ of $50 \mathrm{~mm}$-PBS (pH 7.4), $50 \mu \mathrm{l}$ of $3 \mathrm{mm-EDTA}$ and $50 \mu \mathrm{l}$ of $3 \mathrm{mM}$-xanthine were added to each sample and the mixture was incubated for $10 \mathrm{~min}$ at $24^{\circ} \mathrm{C}$. XO activity was assayed as uric acid production, based on the increase in absorbance at $286 \mathrm{~nm}$. The enzyme activity was expressed as $\mathrm{mU} / \mathrm{mg}$ protein, where one unit corresponds to the formation of $1 \mu \mathrm{mol}$ uric acid per min.

Protein determination. Protein content in the supernatant fractions was estimated by the Bio-Rad DC protein assay kit (Bio-Rad, Segrate, Milan, Italy).

\section{Glutathione reductase $1 \mathrm{mRNA}$ levels in the cerebral cortex}

Total RNA from the cerebral cortex, collected in RNA later buffer (Qiagen, Milan, Italy), was extracted using the RNeasy Midi kit with DNase step (Qiagen), according to the manufacturer's instructions. The purity and quality were checked spectrophotometrically (NanoDrop ${ }^{\mathrm{TM}} 1000$ Spectrophotometer; Thermo Scientific, Rodano, Milan, Italy). For first-strand cDNA synthesis, $1 \mu \mathrm{g}$ of total RNA from each sample was reverse-transcribed using 100 units of RT SuperScript II (Life Technologies, San Giuliano, Milan, Italy) and $1 \mathrm{X}$ random hexamers (Roche Diagnostics, Monza, Italy). Primers to amplify the GR gene were designed on the basis of the sequences reported by the UNIGENE database for the rat (Rn.19721 for GR). Primer sequences are available on request. Each gene was co-amplified together with $\beta$-actin as a control. The PCR was carried out as previously described $^{(33)}$. The PCR products were separated on agarose $(1.6 \%)$ gel. The amplified products were photographed with a digital camera and the intensity of the bands was analysed with Quantity-One software (Bio-Rad, Segrate, Milan, Italy). 
The relative amount of mRNA in the samples was normalised using $\beta$-actin co-amplified as internal standard.

\section{Statistics}

Unless specifically indicated, the results are expressed as mean values with their standard errors, and all data were analysed using one-way ANOVA ( $P$ level fixed at 0.05).

\section{Results}

Antioxidant content of the oils

The antioxidant composition of the olive oils employed is shown in Table 1.

\section{Animal growth}

During the 12-month treatment with high fat, the animals became obese, as it could be expected. At the beginning of the experiment, the average weight was 547.5 (SEM 23.8) g for MO, 552.9 (SEM 15.8) $\mathrm{g}$ for $\mathrm{H}-\mathrm{EVOO}$ and 544.3 (SEM 14.6) $\mathrm{g}$ for L-EVOO rats; at the end, the values were increased to 667.8 (SEM 34.4) g, 710.0 (SEM 24.6) $\mathrm{g}$ and 739.7 (SEM 22.0) g, respectively. No differences were found among the three growth curves.

\section{Behaviour}

No differences in motor or exploratory activity, learning or motor coordination were found among the groups, as shown in Fig. 1. The hole board test performed at 2, 20, 34 and 50 weeks of treatment showed a progressive decrease in the number of movements of the animals on the board, similar for all the groups (Fig. 1(a)). The differences $v$. the first session were significant for all groups at sessions 3 and $4(P<0.05)$. This effect was possibly due to increased body weight and the resulting cumbersomeness in moving rather than to a decrease in interest in surroundings, as indicated by the fact that no significant differences were found in the number of times that the animals explored the holes

Table 1. Antioxidant composition of the study olive oils $(\mathrm{mg} / \mathrm{kg}$ ) (Mean values and standard deviations of three independent determinations)

\begin{tabular}{|c|c|c|c|c|}
\hline \multirow[b]{2}{*}{ Components (mg/kg) } & \multicolumn{2}{|c|}{ H-EVOO } & \multicolumn{2}{|c|}{ L-EVOO } \\
\hline & Mean & SD & Mean & SD \\
\hline 3,4-DHPEA & $15 \cdot 00$ & 0.50 & - & - \\
\hline$p$-HPEA & 11.00 & 0.10 & $0 \cdot 10$ & 0.01 \\
\hline 3,4-DHPEA-EDA & $346 \cdot 70$ & 3.50 & $1 \cdot 10$ & 0.02 \\
\hline$p$-HPEA-EDA & 81.90 & $1 \cdot 10$ & 1.70 & 0.01 \\
\hline (+)-1-Acetoxipinoresinol & $17 \cdot 00$ & 0.02 & 1.50 & 0.01 \\
\hline (+)-Pinoresinol & $32 \cdot 30$ & $0 \cdot 10$ & $2 \cdot 00$ & 0.02 \\
\hline 3,4-DHPEA-EA & 214.90 & $2 \cdot 10$ & 2.90 & 0.03 \\
\hline$\alpha$-Tocopherol & $131 \cdot 20$ & $1 \cdot 10$ & $129 \cdot 10$ & $1 \cdot 10$ \\
\hline
\end{tabular}

H-EVOO, extra-virgin olive oil rich in natural antioxidants; L-EVOO, extra-virgin olive oil poor in natural antioxidants; 3,4-DHPEA, hydroxytyrosol; $p$-HPEA, tyrosol; 3,4-DHPEA-EDA, 3,4 dialdehyde form of elenoic acid linked to hydroxytyrosol; p-HPEA-EDA (oleocanthal), dialdehyde form of elenoic acid linked to tyrosol; 3,4-DHPEA-EA, hydroxytyrosol esters of elenoic acid or oleuropein. over time (Fig. 1(b)). In the multiple Y-maze, assessed once at treatment week 44 , the total space $(\mathrm{cm})$ covered by the animals over a period of $3 \mathrm{~min}$ was not different among groups (263 (SEM 38) for MO, 284 (SEM 44) for H-EVOO, 304 (SEM 56) for L-EVOO). The social learning test was used to evaluate animals' memory function throughout the treatment without using a conditioning step. Over time, all the animals appeared to lose interest in socialisation, as shown by the reduction in time spent exploring the unfamiliar newcomer rat (Fig. 1(c)). A tendency towards age-related memory impairment was indicated in all groups by an increase in the time spent exploring familiar rats, which, however, was not statistically significant (Fig. 1(d)). The fear conditioning test was performed once, close to the end of the experiment (starting on treatment week 53). No difference in freezing time was found among groups either after $3 \mathrm{~d}$ (Fig. 1(e)) or at any of the later times (data not shown). This result in the fear conditioning test was not affected by differences in pain threshold among the groups. In fact, the paw-pressure pain threshold, as measured with the Randall and Selitto test, tended to increase with increasing age, but this tendency was the same for all three experimental groups $(P<0.05$; sessions 3 and $4 v$. session 1 for all the groups; data not shown). Motor coordination was assayed with the rotarod test, performed once, on treatment week 38. Rats from the H-EVOO group were able to maintain equilibrium on the rotarod for a longer average time $(+42 \%)$ than MO rats (Fig. 1(f)). An increase of $11 \%$ over the MO group was also shown by the L-EVOO rats. However, due to high variability, neither of these differences was statistically significant.

Anxiety-related behaviour was assessed with the lightdark preference test, without applying any aversive stimulus, and was performed once on treatment week 41. As shown in Fig. 2(a), the step-through latency was significantly increased $(+50 \% ; P<0.05)$ in the $\mathrm{H}-\mathrm{EVOO}$ group as compared with both the MO and the L-EVOO groups. The total time spent in the lighted chamber also tended to increase in the H-EVOO group (29 (SEM 6.8) $\mathrm{s}$; $+39 \%$ compared with MO (17.7 (SEM 5.7) s); $+41 \%$ compared with L-EVOO (17.3 (SEM 5.4) s)) (Fig. 2(b)). The number of crossings between the compartments was 1.8 (SEM $0 \cdot 3$ ) for all groups.

\section{Markers of oxidative damage and oxidative stress-related enzymes}

Markers of oxidative damage on DNA or lipids did not vary significantly among the groups in the cerebral cortex, nor did the activities of GPx, SOD and XO. The levels of total glutathione and GSH and of GSSG were also unchanged among the experimental groups (Table 2).

However, the activity of GR was decreased ( $-20 \%$; $P<0.05$ v. MO; Fig. 3) in the H-EVOO group as compared with controls, and a negative correlation between GR activity and step-through latency in the light-dark preference test ( $R \quad 0.62 ; P<0.05$ ) was found. The expression of the GR gene in the cerebral cortex was also significantly reduced in the H-EVOO group ( $-25 \% ; P<0.05$ v. MO; Fig. 3). 

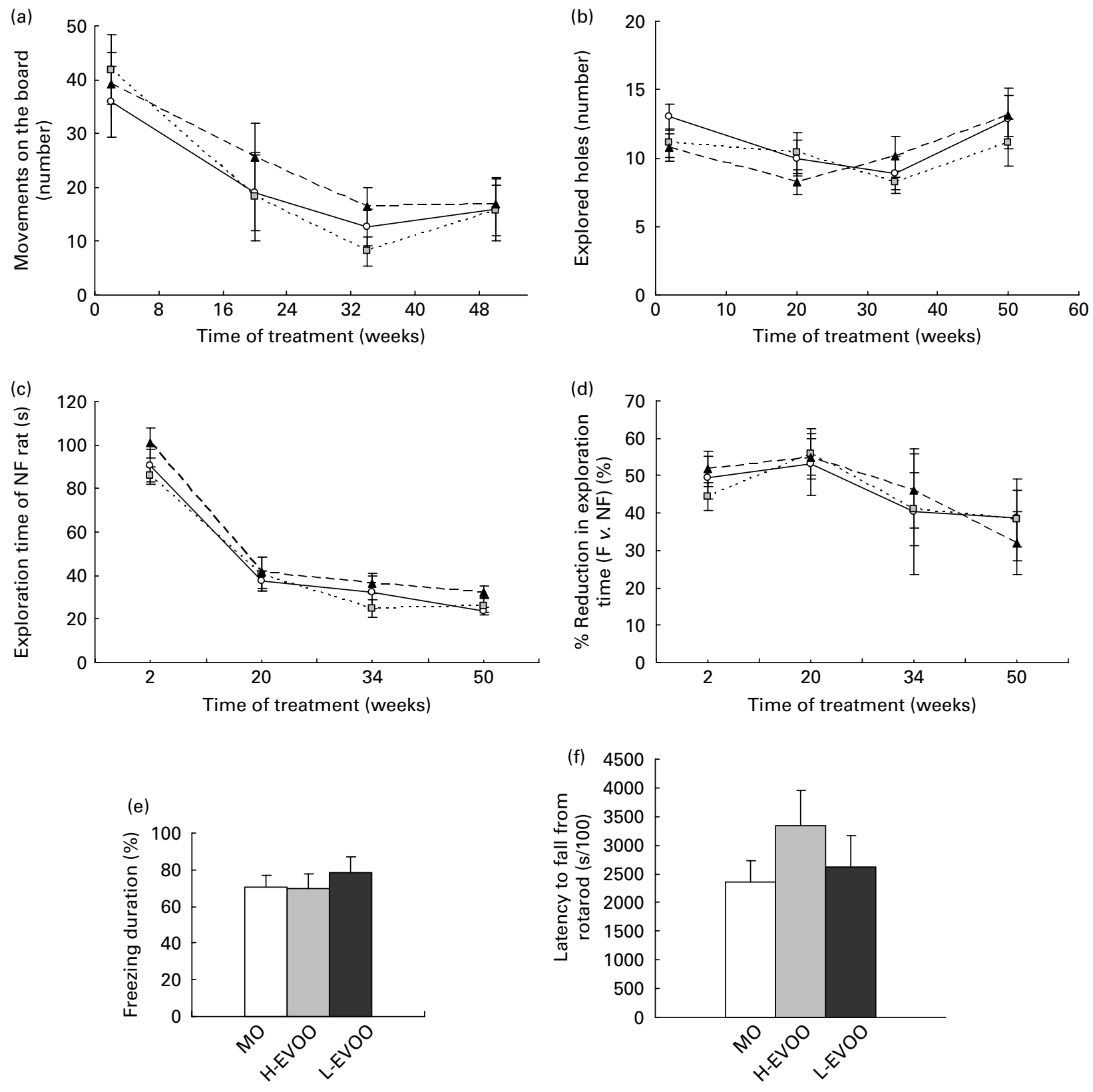

Treatment

Treatment

Fig. 1. Cognitive and motor behaviour: spontaneous motility (a) and exploratory activity (b) in the rat hole-board test. (a) Number of movements on the board was measured at treatment weeks 2, 20,34 and 50. (-O-), Maize oil (MO) group; (-- ---), extra-virgin olive oil rich in natural antioxidants (H-EVOO) group; $(--\mathbf{\Delta -}-)$, extra-virgin olive oil poor in natural antioxidants (L-EVOO) group. Values are means of ten animals, with standard errors represented by vertical bars. The decrease in spontaneous motility in comparison with week 2 was statistically significant for all the groups at weeks 34 and 50 ( $P<0.05$; ANOVA). (b) Number of explored holes. Values are means of ten animals, with standard errors represented by vertical bars. Memory tests: social learning (c, d) and contextual fear conditioning test (e). The social learning test was repeated for four sessions, at treatment weeks 2, 20, 34 and 50. (c) Exploration time in seconds of an unfamiliar rat (NF) was measured on day 1 (see Methods and materials). Values are means of ten animals, with standard errors represented by vertical bars. The decrease in social interaction compared with session 1 was significant for all the groups at sessions 2,3 and $4(P<0.05$; ANOVA). (d) Learning expressed as percentage reduction of the exploration time of the familiar rat (F) $v$. NF. Lower values indicate deterioration of memory function. Values are means of ten animals, with standard errors represented by vertical bars. (e) The fear conditioning test was performed at treatment week 53 on a pre-designated subset of each group: nine animals (MO group); nine animals (H-EVOO group); six animals (L-EVOO group). Each rat was placed in the apparatus on the third day after the conditioning session, and left undisturbed for $180 \mathrm{~s}$. During this time, the total accumulated freezing time in seconds was measured and expressed as percentage of the total $180 \mathrm{~s}$. Values are means, with standard errors represented by vertical bars. (f) Motor coordination in the rotarod test. Time to fall from an accelerating rotarod was measured at treatment week 38 from a pre-designated subset of each group: ten animals (MO group); ten animals (H-EVOO group); eight animals (L-EVOO group). Values are means, with standard errors represented by vertical bars. 


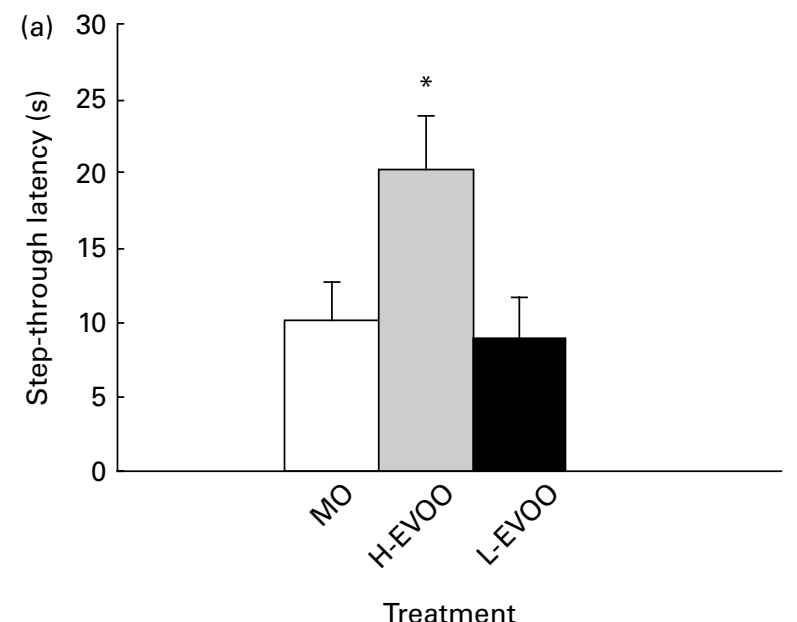

(b)

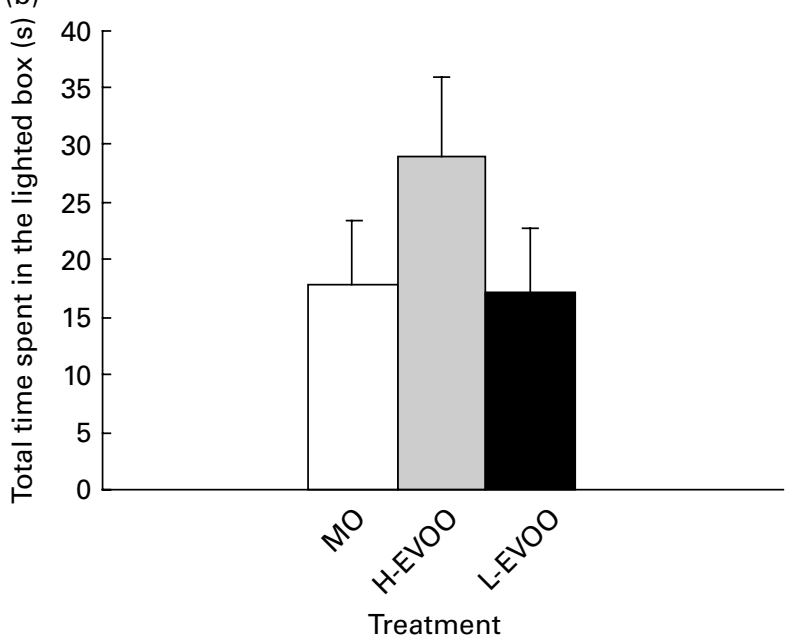

Fig. 2. Light-dark box test. The step-through latency (a) and the total time spent in the light (b) were measured at treatment week 41 from a predesignated subset of each group: ten animals in the maize oil (MO) group; ten animals in the extra-virgin olive oil rich in natural antioxidants (H-EVOO) group; eight animals in the extra-virgin olive oil poor in natural antioxidants (L-EVOO) group. Values are means, with standard errors represented by vertical bars. * Mean value was significantly different from those of the MO and L-EVOO groups $(P<0.05)$.

\section{Discussion}

The aim of the present study was to verify whether extravirgin olive oil, rich in phenolic antioxidants, has the potential to ameliorate some functional and biochemical alterations brought about by brain ageing. In particular, we were interested in verifying whether these compounds could improve cognitive and motor functions in aged animals subjected to a high-fat high-energy regimen. In fact, other antioxidants, such as the natural stilbene resveratrol, have been reported to improve motor coordination in aged mice fed a high-fat diet by Baur et al. ${ }^{(34)}$ and Pearson et al. ${ }^{(35)}$.

Under the present experimental conditions, extra-virgin olive oil provided a significant amount of phenolic antioxidants (about $2 \mathrm{mg}$ total phenolic compounds/rat per d, of which 3,4-DHPEA and oleuropein represented about $30 \%$ ). The calculated amount of vitamin $\mathrm{E}$ provided by both olive oils was about $0.9 \mathrm{mg} / \mathrm{rat}$ per $\mathrm{d}$, in addition to the basal level of vitamin $\mathrm{E}$ contained in the standard rat diet $(0.4 \mathrm{mg} / \mathrm{rat}$ per d). We have previously shown that this treatment was associated with decreased age-related ulcerative dermatitis and with a tendency towards reduced mortality during ageing in the H-EVOO group ${ }^{(1,21)}$, and we were interested in studying whether some beneficial effects could also be detected in the central nervous system. At the end of the treatment, parameters related to oxidative stress, such as DNA damage, lipid peroxidation, GSH and GSSG levels, and GPx, SOD and XO activity in the cerebral cortex, were not different among the groups. It is important to take into account that in the present study we employed a high-fat diet, based on the high lipid content prevailing in the human diet of many countries, and high-fat, high-energy diets are associated with increased oxidative stress. In the rat, increased protein and lipid oxidation has been reported in brain regions upon administration of diets containing higher amounts of lipids ${ }^{(36,37)}$. In the cerebral cortex, a high-fat diet induced increased reactive oxygen species (ROS) and inflammation markers ${ }^{(38)}$. Thus, it is possible that the pro-oxidant conditions associated with the high-fat regimen overwhelm the possible beneficial effects of olive oil phenolic antioxidants.

Of the employed battery of behavioural tests, only some were performed throughout the treatment, in order to avoid overstimulation of the animals. The complete set of tests was performed at the end of the experiment, when the animals were old, as one objective of the present study was to verify the effects on ageing, and when the probability of seeing some effects was presumably maximal, at least in terms of length of the treatment. Contrary to our expectations, no significant differences in motor or exploratory activity, learning or motor coordination were found among the groups. Although the animals did not have reduced exploratory drive, over time they did show reduced motility, probably because of their increased body weight. This last result is in agreement with previous literature reports showing a decrease in locomotor activity in aged animals ${ }^{(39)}$. With progressing age, all the animals also showed less interest in socialisation as assayed by the social learning test. In fact, the interaction time with an unfamiliar rat declined from values similar to young animals ${ }^{(40)}$ at the beginning of the experiment to values $70 \%$ lower at the end. A tendency towards a decline in memory in the older animals was also seen with the social learning test. The fact that these differences did not reach significance might be due to a progressive decrease in exploration times of both the unfamiliar and the familiar rat, which hampered the potency of the test. Prediger et al. ${ }^{(41)}$ reported an age-related disruption in the rat social recognition ability along with a less drastic (about 50\%) reduction in exploration of the unfamiliar rat than under our experimental conditions. This could be related to the increased weight of our high fat-fed animals which rendered them less mobile, although we do not have direct experimental evidence to substantiate this hypothesis. In agreement with the data obtained with the social learning test, the employed treatments did not affect cognitive functions in the fear conditioning test either.

The pain threshold increased with age in agreement with previous findings ${ }^{(39)}$ but was not modified by the treatments. Thus, the absence of differences among groups in the fear conditioning test was not confounded by differences in pain sensitivity. 
Table 2. Oxidative damage-related parameters in the cerebral cortex* (Mean values with their standard errors)

\begin{tabular}{|c|c|c|c|c|c|c|}
\hline & \multicolumn{2}{|c|}{ MO } & \multicolumn{2}{|c|}{ H-EVOO } & \multicolumn{2}{|c|}{ L-EVOO } \\
\hline & Mean & SEM & Mean & SEM & Mean & SEM \\
\hline DNA strand breaks (\% DNA in tail) & $6 \cdot 27$ & 0.52 & $6 \cdot 13$ & 0.49 & 6.52 & 0.95 \\
\hline DNA FPG sites (\% DNA in tail) & $15 \cdot 44$ & 1.85 & 13.66 & 1.66 & $15 \cdot 14$ & $2 \cdot 18$ \\
\hline MDA (nmol/mg protein) & 1.91 & 0.31 & 1.54 & 0.30 & 1.49 & 0.28 \\
\hline GSH (nmol/mg protein) & $15 \cdot 54$ & 2.38 & 16.91 & $2 \cdot 77$ & 14.82 & $2 \cdot 62$ \\
\hline GSSG (nmol/mg protein) & 0.36 & 0.07 & 0.36 & 0.08 & 0.42 & 0.09 \\
\hline GSH:GSSG & $49 \cdot 51$ & 14.94 & 59.01 & $17 \cdot 70$ & 39.57 & 6.06 \\
\hline GPx activity (nmol NADPH/min per mg protein) & $20 \cdot 73$ & 3.85 & $22 \cdot 24$ & 2.55 & $21 \cdot 72$ & $2 \cdot 87$ \\
\hline SOD activity (U/mg protein)† & 4.66 & $1 \cdot 13$ & 4.40 & 0.93 & $3 \cdot 33$ & 0.69 \\
\hline $\mathrm{XO}$ activity (mU/mg protein)† & $138 \cdot 00$ & $20 \cdot 90$ & 143.00 & $18 \cdot 50$ & 133.00 & $24 \cdot 60$ \\
\hline
\end{tabular}

MO, maize oil; H-EVOO, extra-virgin olive oil rich in natural antioxidants; L-EVOO, extra-virgin olive oil poor in natural antioxidants; FPG, formamidopyrimidine glycosylase; MDA, malonyldialdehyde; GSH, reduced glutathione; GSSG, oxidised glutathione; GPx, glutathione peroxidase; SOD, superoxide dismutase; $X O$, xanthine oxidase.

* Values are expressed for ten animals for DNA strand breaks and FPG sites, and for five or six animals for the other parameters.

†For definitions of units, see Methods and materials.

Finally, motor coordination, evaluated with the rotarod, tended to be better in the H-EVOO group, but the difference did not reach statistical significance due to high variability. This lack of effect can be explained by the lower dose of antioxidants (about $4 \mathrm{mg}$ total phenolic compounds $/ \mathrm{kg}$ ) used in the present study compared with those employed in other studies where natural antioxidants have shown protective effects on cognitive and motor behaviour. For example, the studies by Joseph et al. ${ }^{(8)}$ employed extracts of fruit or vegetables yielding final Trolox equivalents about 10-fold higher than in the standard diet. The most effective dose of resveratrol in the study by Baur et al. ${ }^{(34)}$ was $22.4 \mathrm{mg} / \mathrm{kg}$. Thus, aside from the different biological activities and kinetics of the various compounds involved, it is possible that the phenol doses in the present study were not high enough to obtain stronger effects. On the other hand, these are the maximal doses that can be attained by administering natural oils and not adding phenolic antioxidants as supplements.

One interesting effect of olive oil phenols was a decreased step-through latency in the light-dark preference test, one of the most commonly used rodent models of anxiety ${ }^{(42)}$. Under our experimental conditions, the H-EVOO-treated animals showed less tendency to enter and to remain in the dark compartment than the other groups. This effect could be attributed to reduced anxiety in the H-EVOO group and not to differences in motor or exploratory activity, which were not modified by the treatments. However, in the lightdark box test no statistically significant difference was found in the total time spent in the lighted compartment, although this parameter was increased in the H-EVOO group, and there was no difference among groups in the number of crossings between the two compartments. This observation, along with a lack of inter-group differences in the fear conditioning, hole-board and multiple Y maze tests, which are also sensitive to changes in anxiety, indicates that the effect of olive oil phenols on anxiety-like behaviour is limited. Apparently, at least under our experimental conditions, this effect is essentially a reduction of the initial drive of the animals to escape into the dark compartment. It is very likely that this effect was due to the phenol content of olive oil, as it was not detected in the animals treated with the low-phenol olive oil.
An anxiety-lowering activity in aged animals has been shown using the elevated plus maze by Viggiano et al. ${ }^{(9)}$ upon prolonged treatment with an apple-rich diet, presumably due to the antioxidant content of the fruit. It has also been shown that the polyphenol chlorogenic acid exerts anxiolytic effects coupled with antioxidant activity in mice ${ }^{(43)}$. A link between anxiety-related behaviour and oxidative stress has been indicated by some human studies showing that changes in the levels of antioxidants are associated with anxiety disorders ${ }^{(44,45)}$. Such a link has also been found in animal models, which have generated intriguing findings. In a gene expression analysis study, Hovatta et al. ${ }^{(46)}$ proposed a genetic component in the vulnerability to anxiety disorders,

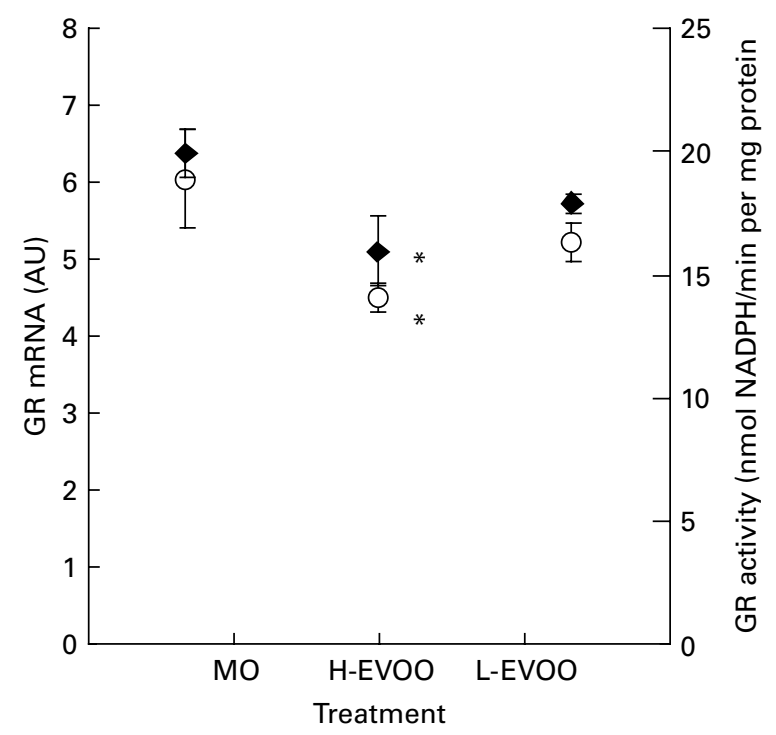

Fig. 3. Glutathione reductase (GR) gene expression (GR mRNA; $O$ ) and GR activity $(\downarrow)$ in the cerebral cortex. The levels of mRNA were calculated as relative to $\beta$-actin and expressed in arbitrary units (AU). The enzymic activity was expressed as nmol oxidised NADPH/min per mg protein. MO, maize oil; $\mathrm{H}-\mathrm{EVOO}$, extra-virgin olive oil rich in natural antioxidants; L-EVOO, extravirgin olive oil poor in natural antioxidants. Values are means of ten animals, with standard errors represented by vertical bars. * Mean value was significantly different from that of the MO group $(P<0.05)$. 
showing a correlation between anxiety and expression of glyoxalase 1 (Glo1) and GR in mouse brain, related to antioxidant enzymic defences. A possible role for these enzymes in anxiety has also emerged from the work of other groups employing different animal models ${ }^{(47)}$; however, in the work of Hovatta the levels of expression and activity of these enzymes decreased when anxiety was low, whereas the opposite was shown by Kromer et al. ${ }^{(47)}$ for Glo1. In agreement with the work of Hovatta et al., we found both reduced GR mRNA and reduced GR activity in the cerebral cortex of the animals displaying a reduced drive to pass into the dark compartment, and a negative correlation between cortical GR activity and step-through latency in the light-dark preference test. These results raise the possibility that modulation of gene expression and activity of GR might have a beneficial effect on anxiety-like behaviour.

It is intriguing that other antioxidant enzymes such as GPx and SOD were not modulated in the rat brain by the treatment with olive oil phenols, and no detectable change in oxidative damage or in the levels of GSH or GSSG was detected. GR converts GSSG generated in reactions catalysed by GPx to its reduced form, which has a major role in protecting the brain against oxidative stress by interacting directly with ROS or by participating in enzyme-catalysed redox cycling reactions ${ }^{(48)}$. However, the present results do not support a direct, straightforward correlation between oxidative stress and anxiety.

In conclusion, the present study indicates that under conditions of prolonged high fat intake, olive oil phenols do not exert major protective actions on motor and cognitive functions of the ageing brain, but they might have an effect on anxiety-associated behaviour, possibly through a modulation of the expression level of GR, a gene involved in the defence mechanisms against oxidative stress. Changes in emotional behaviour are an important aspect of human ageing. The possibility of using antioxidant phenols from olive oil to modulate emotional behaviour will have to be confirmed in other experimental models and animal species.

\section{Acknowledgements}

The present study was supported by the Ministry of Education University and Research (MIUR) project FISR 'Miglioramento delle proprietà sensoriali e nutrizionali di prodotti alimentari di origine vegetale relativi alla prima e alla seconda trasformazione (Improving the nutritional and sensory properties of foods of plant origin for the first and second processing)', by the University of Florence, by the European Union (EU) program FLAVO 2005-513960 and by the EU Network of Excellence in Nutrigenomics, NuGO FOODCT-2004-506360. We thank Andrew R. Collins (University of Oslo, Norway) for kindly providing the enzyme FPG, and Mary Forrest for revising the manuscript.

All experiments were designed and carried out by V. P., M. J., P. D. and L. G., with the exceptions noted below. M. Z., C. L., E. B. and M. L. performed the analyses of malonyldialdehyde levels, SOD and XO activity and GR mRNA. C. G., E. V. and M. N. assisted with design and interpretation of the data obtained with the hole board, social learning, and RandallSelitto tests. M. G., E. B. and C. B. assisted with the design and interpretation of the data obtained with the rotarod, fear conditioning, light-dark box and $\mathrm{Y}$ maze tests. S. E. and M. S. performed the chemical analyses of the oils. G. M. assisted with the design and interpretation of all experiments.

All authors read and approved the final version of the manuscript and declare that there are no actual or potential conflicts of interest.

\section{References}

1. Mecocci P, MacGarvey U, Kaufman AE, et al. (1993) Oxidative damage to mitochondrial DNA shows marked age-dependent increases in human brain. Ann Neurol 34, 609-616.

2. Giovannelli L, Decorosi F, Dolara P, et al. (2003) Vulnerability to DNA damage in the aging rat substantia nigra: a study with the comet assay. Brain Res 969, 244-247.

3. Tian L, Cai Q \& Wei H (1998) Alterations of antioxidant enzymes and oxidative damage to macromolecules in different organs of rats during aging. Free Radic Biol Med 24, $1477-1484$.

4. O'Donnell E, Vereker E \& Lynch MA (2000) Age-related impairment in LTP is accompanied by enhanced activity of stress-activated protein kinases: analysis of underlying mechanisms. Eur J Neurosci 12, 345-352.

5. Mattson MP (2002) Oxidative stress, perturbed calcium homeostasis, and immune dysfunction in Alzheimer's disease. J Neurovirol 8, 539-550.

6. Jenner P \& Olanow CW (1996) Oxidative stress and the pathogenesis of Parkinson's disease. Neurology 47, S161-S170.

7. Youdim KA, Spencer JP, Schroeter H, et al. (2002) Dietary flavonoids as potential neuroprotectants. Biol Chem 383, 503-519.

8. Joseph JA, Shukitt-Hale B, Denisova NA, et al. (1999) Reversals of age-related declines in neuronal signal transduction, cognitive, and motor behavioral deficits with blueberry, spinach, or strawberry dietary supplementation. J Neurosci 19, 8114-8121.

9. Viggiano A, Viggiano A, Monda M, et al. (2006) Annurca apple-rich diet restores long-term potentiation and induces behavioral modifications in aged rats. Exp Neurol 199, 354-361.

10. Pallardó FV, Asensi M, García de la Asunción J, et al. (1998) Late onset administration of oral antioxidants prevents agerelated loss of motor co-ordination and brain mitochondrial DNA damage. Free Radic Res 29, 617-623.

11. Servili M, Selvaggini R, Esposto S, et al. (2004) Health and sensory properties of virgin olive oil hydrophilic phenols: agronomic and technological aspects of production that affect their occurrence in the oil. J Chromatogr A 1054, 113-127.

12. Lavelli V (2002) Comparison of the antioxidant activities of extra virgin olive oils. J Agric Food Chem 50, 7704-7708.

13. Visioli F, Caruso D, Plasmati E, et al. (2001) Hydroxytyrosol, as a component of olive mill waste water, is dose-dependently absorbed and increases the antioxidant capacity of rat plasma. Free Radic Res 34, 301-305.

14. Miro-Casas E, Covas MI, Fito M, et al. (2003) Tyrosol and hydroxytyrosol are absorbed from moderate and sustained doses of virgin olive oil in humans. Eur J Clin Nutr 57, 186-190.

15. Manna C, Galletti P, Cucciolla V, et al. (1997) The protective effect of the olive oil polyphenol (3,4-dihydroxyphenyl)-ethanol counteracts reactive oxygen metabolite-induced cytotoxicity in Caco-2 cells. J Nutr 127, 286-292.

16. Aruoma OI, Deiana M, Jenner A, et al. (1998) Effect of hydroxytyrosol found in extra virgin olive oil on oxidative DNA damage and on low-density lipoprotein oxidation. J Agric Food Chem 46, 5181-5187.

17. Visioli F, Galli C, Plasmati E, et al. (2000) Olive phenol hydroxytyrosol prevents passive smoking-induced oxidative stress. Circulation 102, 2169-2171. 
18. Visioli F, Poli A \& Gall C (2002) Antioxidant and other biological activities of phenols from olives and olive oil. Med Res Rev 22, 65-75.

19. Schaffer S, Podstawa M, Visioli F, et al. (2007) Hydroxytyrosolrich olive mill wastewater extract protects brain cells in vitro and ex vivo. J Agric Food Chem 55, 5043-5049.

20. Solfrizzi V, Panza F, Torres F, et al. (1999) High monounsaturated fatty acids intake protects against age-related cognitive decline. Neurology 52, 1563-1569.

21. Jacomelli M, Pitozzi V, Zaid M, et al. (2009) Dietary extra-virgin olive oil rich in phenolic antioxidants and the aging process: long-term effects in the rat. $J$ Nutr Biochem (epublication ahead of print version 13 April 2009).

22. Grigg D (1999) The fat of the land: a geography of oil and fat consumption. Geojournal 48, 259-268.

23. Selvaggini R, Servili M, Urbani S, et al. (2006) Evaluation of phenolic compounds in virgin olive oil by direct injection in high-performance liquid chromatography with fluorometric detection. J Agric Food Chem 54, 2832-2838.

24. Psomiadou E \& Tsimidou M (1998) Simultaneous HPLC determination of tocopherols, carotenoids and chlorophylls for monitoring their effect on virgin olive oil oxidation. J Agric Food Chem 46, 5132-5138.

25. Ambrogi Lorenzini C, Bucherelli C, Falchini S, et al. (1990) The rat's right-left preference during free exploration of a multiple Y-maze. Physiol Behav 48, 175-177.

26. Leighton GE, Rodriguez RE, Hill RG, et al. (1988) к-Opioid agonists produce antinociception after i.v. and i.c.v. but not intrathecal administration in the rat. BrJ Pharmacol 93, 553-560.

27. Mondadori C, Preiswerk G \& Jaekel J (1992) Treatment with a $\mathrm{GABA}_{\mathrm{B}}$ receptor blocker improves the cognitive performance of mice, rats and rhesus monkey. Pharmacol Comm 2, 93-97.

28. Sacchetti B, Ambroqi LC, Baldi E, et al. (1999) Memorization of contextual and CS conditioned fear response (freezing) in a one-trial acquisition paradigm. Arch Ital Biol 137, 235-248.

29. Ambrogi LC, Bucherelli C \& Giachetti A (1986) Some factors influencing conditioned and spontaneous behavior of rats in the light-dark box test. Physiol Behav 36, 97-101.

30. Mateos R, Lecumberri E, Ramos S, et al. (2005) Determination of malondialdehyde (MDA) by high-performance liquid chromatography in serum and liver as a biomarker for oxidative stress. Application to a rat model for hypercholesterolemia and evaluation of the effect of diets rich in phenolic antioxidants from fruits. J Chromatogr B Analyt Technol Biomed Life Sci 827, 76-82

31. Paglia DE \& Valentine WN (1967) Studies on the quantitative and qualitative characterization of erythrocyte glutathione peroxidase. J Lab Clin Med 70, 158-169.

32. Carlberg I \& Mannervik B (1985) Glutathione reductase. Methods Enzymol 113, 484-490.

33. Luceri C, Caderni G, Sanna A, et al. (2002) Red wine and black tea polyphenols modulate the expression of cycloxygenase-2, inducible nitric oxide synthase and glutathione-related enzymes in azoxymethane-induced f344 rat colon tumors. J Nutr 132, 1376-1379.

34. Baur JA, Pearson KJ, Price NL, et al. (2006) Resveratrol improves health and survival of mice on a high-calorie diet. Nature 444, 337-342.

35. Pearson KJ, Baur JA, Lewis KN, et al. (2008) Resveratrol delays age-related deterioration and mimics transcriptional aspects of dietary restriction without extending life span. Cell Metab 8, 157-168.

36. Souza CG, Moreira JD, Siqueira IR, et al. (2007) Highly palatable diet consumption increases protein oxidation in rat frontal cortex and anxiety-like behavior. Life Sci 81, 198-203.

37. Fachinetto R, Burger ME, Wagner C, et al. (2005) High fat diet increases the incidence of orofacial dyskinesia and oxidative stress in specific brain regions of rats. Pharmacol Biochem Behav 81, 585-592.

38. Zhang X, Dong F, Ren J, et al. (2005) High dietary fat induces NADPH oxidase-associated oxidative stress and inflammation in rat cerebral cortex. Exp Neurol 191, 318-325.

39. Altun M, Bergman E, Edstrom E, et al. (2007) Behavioral impairments of the aging rat. Physiol Behav 92, 911-923.

40. Ghelardini C, Galeotti N, Gualtieri F, et al. (1997) 3- $\alpha$-Tropanyl 2-(4-Cl-phenoxy) butyrate (SM-21): a review of the pharmacological profile of a novel enhancer of cholinergic transmission. CNS Drug Reviews 3, 346-362.

41. Prediger RD, Batista LC \& Takahashi RN (2005) Caffeine reverses age-related deficits in olfactory discrimination and social recognition memory in rats. Involvement of adenosine A1 and A2A receptors. Neurobiol Aging 26, 957-964.

42. Holmes A, Iles JP, Mayell SJ, et al. (2001) Prior test experience compromises the anxiolytic efficacy of chlordiazepoxide in the mouse light/dark exploration test. Behav Brain Res 122, 159-167.

43. Bouayed J, Rammal H, Dicko A, et al. (2007) Chlorogenic acid, a polyphenol from Prunus domestica (Mirabelle), with coupled anxiolytic and antioxidant effects. $J$ Neurol Sci 262, 77-84.

44. Ersan S, Bakir S, Erdal EE, et al. (2006) Examination of free radical metabolism and antioxidant defence system elements in patients with obsessive-compulsive disorder. Prog Neuropsychopharmacol Biol Psychiatry 30, 1039-1042.

45. Atmaca M, Kuloglu M, Tezcan E, et al. (2008) Antioxidant enzyme and malondialdehyde levels in patients with social phobia. Psychiatry Res 159, 95-100.

46. Hovatta I, Tennant RS, Helton R, et al. (2005) Glyoxalase 1 and glutathione reductase 1 regulate anxiety in mice. Nature 438, 662-666.

47. Kromer SA, Kessler MS, Milfay D, et al. (2005) Identification of glyoxalase-I as a protein marker in a mouse model of extremes in trait anxiety. $J$ Neurosci 25, 4375-4384.

48. Cooper AJ \& Kristal BS (1997) Multiple roles of glutathione in the central nervous system. Biol Chem 378, 793-802. 